\title{
INTRUSIVE EXPERIENCES IN POST-TRAUMATIC STRESS DISORDER: TREATMENT RESPONSE INDUCES CHANGES IN THE EFFECTIVE CONNECTIVITY OF THE ANTERIOR INSULA
}

\author{
Abbreviated title: \\ Insular Cortex Influence on Intrusivity
}

\section{Author list:}

Arnaud Leroy ${ }^{1,2,3^{*}}$, Etienne Very ${ }^{4,5}$, Philippe Birmes ${ }^{5}$, Sébastien Szaffarczyk ${ }^{1}$, Renaud Lopes ${ }^{6,7}$, Cécile Faure ${ }^{1}$, Stéphane Duhem ${ }^{2,3}$, Pierre Grandgenèvre ${ }^{2}$, Frédérique Warembourg ${ }^{2}$, Guillaume Vaiva ${ }^{1,2,3}$, Renaud Jardri ${ }^{1,8}$

\section{Affiliations :}

1. Univ Lille, INSERM, CHU Lille, Lille Neuroscience \& Cognition Centre (U-1772), Plasticity \& SubjectivitY team, CURE platform, 59000 Lille, France

2. CHU Lille, Fontan Hospital, General Psychiatry Dpt., 59037 Lille cedex, France

3. Centre National de Ressources et Résilience pour les psychotraumatismes (CN2R Lille - Paris), 59000 Lille, France

4. CHU Toulouse, Purpan Hospital, Psychiatry Department, 31059 Toulou se Cedex, France

5. ToNIC, Toulouse Neurolmaging Center, INSERM U-1214, UPS, France

6. Univ Lille, INSERM, CHU Lille, Lille Neuroscience \& Cognition Centre (U-1772), Degenerative \& Vascular Cognitive Disorders team, 59000 Lille, France

7. Univ Lille, CNRS, INSERM, CHU Lille, Institut Pasteur de Lille, US 41 - UMS 2014 - PLBS, 59000 Lille, France

8. CHU Lille, Fontan Hospital, Child \& Adolescent Psychiatry Dpt., 59037 Lille cedex, France

Corresponding Author: *, arnaud.leroy@chru-lille.fr

Number of display items: 3 figures $/ 2$ tables +8 suppl. items

\section{Number of words: 3629}

Statement of interest: AL is consultant for Kinnov Therapeutics. RJ has been invited to scientific meetings, and boards with compensation, by Lundbeck, Janssen and Otsuka. None of these links of interest are related to the present work. 


\section{ABSTRACT (250 words)}

Background: One of the core features of posttraumatic stress disorder (PTSD) is reexperiencing the trauma. The anterior insula (Al) was proposed to play a crucial role in these intrusive experiences. However, the dynamic function of the $\mathrm{Al}$ in reexperiencing trauma, as well as its putative modulation by effective therapy, still need to be specified.

Methods: Thirty PTSD patients were enrolled and exposed to traumatic memory reactivation therapy. Resting-state fMRI scans were acquired before and after treatment. To explore Al directed influences over the rest of the brain, we referred to a mixed-model using pre/post Granger causality analysis seeded on the Al as a within-subject factor and treatment response as a between-subject factor. To further identify correlates of reexperiencing trauma, we investigated how intrusive severity affected: (i) causality maps and (ii) the spatial stability of other intrinsic brain networks.

Results: We observed dynamic changes in Al effective connectivity in PTSD patients. Many withinand between-network causal paths were found to be less influenced by the Al after effective therapy. Insular influences were found positively correlated with flashback severity, while reexperiencing was linked with a stronger default mode network (DMN) and more unstable central executive network (CEN) connectivity.

Conclusions: We showed that directed changes in Al signaling to the DMN and CEN at rest may underlie the degree of intrusive symptoms in PTSD. A positive response to treatment further induced changes in network-to-network anticorrelated patterns. Such findings may guide targeted neuromodulation strategies in PTSD patients not suitably improved by conventional treatment.

\section{Key-words:}

Post-Traumatic Stress Disorder; Treatment response; Effective connectivity; Salience network; fMRI; Reexperiencing 


\section{INTRODUCTION}

Posttraumatic stress disorder (PTSD) is a disabling condition that can be triggered by terrifying events that have the potential to disrupt life, such as interpersonal violence, combat, life-threatening accidents or disasters, as well as global pandemics (1). PTSD may lead to chronic psychiatric or addictive morbidities, loss of normal daily functioning, and increased risk of suicide (2). This disorder usually induces intrusive symptoms (i.e., distressing recollections of the event, including flashbacks and nightmares, often called "re-experiences"), persistent avoidance of stimuli associated with the trauma, negative alterations in cognitions or mood, and hyperarousal (3). "Re-experiencing" is considered central in the pathophysiology of PTSD, despite some similarities with other intrusive thoughts observed transdiagnostically, such as hallucinations, ruminations or persistent worries (47). Even if this research field is prolific, it still lacks a common neurofunctional signature for intrusive experiences that adequately circumscribes the underlying mechanisms of PTSD.

Brain-wide dysconnectivity has been suggested at the root of several psychiatric disorders, and key structures have picked the interest of PTSD scientists. Among the candidate nodes, the anterior insula (AI) was identified as one of the major connector hubs in the brain (8). This structure has been implicated in a large variety of functions, ranging from feelings representation to bodily and selfawareness (9). The Al receives convergent inputs from multiple sensory modalities, including the auditory and visual systems (10-14), and converging evidence supports its involvement in simultaneous attention to multisensory events $(15,16)$. The Al was also proposed to tag salient endogenous and external information and further reallocate attentional resources towards them (17), making it a central element of the "salience network" (SN).

On a more pathological side, hyperarousal and re-experiencing were shown to be associated with increased activity in the bilateral Al and connected limbic structures, such as the amygdala (18) or the hippocampus (19). These symptoms were also associated with an increased functional connectivity of the SN network as a whole (20). Interestingly, aberrant SN effective connectivity was 
also proven involved in the process of switching from a state of unconstrained rest to one of experiencing hallucinatory events in schizophrenia patients $(21,22)$, reinforcing the idea that this network may govern intrusive experiences in general.

In this vein, the $S N$ is thought to tightly control the balance between various intrinsic networks and to swiftly move from rest to task-based actions and vice-versa, a theory that has been conceptualized as the tripartite model $(17-21,23)$. According to this framework, the SN may drive commonly observed anticorrelated patterns between the default mode network (DMN, underlying self-referential processes) and the central executive network (CEN, involved in cognitive control and decision making), an interaction already shown to be impaired in PTSD. For instance, aberrant increases in SN and decreased DMN functional connectivity were described in PTSD patients (20), whereas a surge in connectivity strength of the CEN was reported to be associated with intrusiveness (24). Though these findings are encouraging, some gaps remain, such as the exact links between Al dysconnectivity and other biological theories of PTSD.

At the microscale level, we indeed know that $\mathrm{SN}$ activation is mediated by the B-noradrenergic system. In particular, activation of the locus coeruleus, the main source of epinephrine secretion in the brain, was found to be involved in SN activation (25). The administration of drugs increasing the epinephrine level has also been linked with SN activation $(26,27)$, whereas the administration of propranolol, a noradrenergic beta-receptor blocker, deactivates the SN (28). Finally, a genetic study revealed that only carriers of a common functional deletion in ADRA2B, a gene coding for the $\alpha 2 b-$ adrenoreceptor, displayed increased phasic amygdala responses under stress (29), again supporting the existence of an association between salience responses and B-noradrenergic regulation.

Interestingly, trauma memory reactivation therapy using propranolol (considered a putative reconsolidation blocker) was found to reduce symptom severity in PTSD (30). Referring to a similar randomized controlled trial (RCT) design, the present study intends to bring new insight to the dynamic role of the SN in PTSD and, more specifically, to circumscribe the directed influence of $\mathrm{Al}$ 
over the rest of the brain as a function of treatment response. We hypothesize that effective therapy can modulate the effective connectivity of $\mathrm{Al}$ and that these plastic changes correlate with a reduction in trauma re-experiencing symptoms. In reference to the tripartite model, we also expect this downgrading in intrusiveness to be linked to changes in DMN and CEN spatial stability, demonstrating a brain-wide reallocation of cognitive resources in PTSD patients who respond to treatment.

\section{MATERIALS AND METHODS}

\section{Population}

Thirty patients with a primary diagnosis of PTSD according to DSM-IV-TR criteria (Structured Clinical Interview for DSM-IV, PTSD module) provided written informed consent to participate in the research (Table 1, Suppl. Table 1). They were all taken from a RCT testing for the efficacy of traumatic memory reactivation under the influence of propranolol versus placebo one week posttreatment. This trial received approval from an ethics committee (CPP 2009-012976-29) and was registered on clinicaltrials.gov (NCT01713556). The main results for this clinical trial are presented elsewhere (31).

Participants waiting for treatment were randomly and blindly allocated to two experimental groups (1:1 ratio): (i) a "traumatic memory reactivation therapy + propranolol" group, and (ii) a "traumatic memory reactivation therapy + placebo" group. Propranolol or placebo was administered 90-minutes before the memory reactivation session, performed once a week for 6 consecutive weeks. The Posttraumatic Stress Disorder Checklist Scale - PCL-S (32) was used to quantify symptom severity and assess treatment response. Because we were interested in intrusive symptoms, we focused on the item sum of PCL-S Q1-to-Q5 (32). Assessment was made before treatment (V1) and 
one week after the end of the treatment (V7). The response to memory reactivation (with or without propranolol) in the whole sample (sometimes referred to as therapy in this paper for the sake of simplicity (33)) was considered positive for at least a $33 \%$ decrease $(34,35)$ in the PCL-S Q1-to-Q5 score compared to baseline (this threshold is commonly referred to as the first significant level of response also called "poor response").

\section{$\underline{\text { MRI acquisition and data preprocessing }}$}

Patients underwent two MRI sessions at rest with their eyes closed (at V1 and V7) on a 3T Philips Achieva scanner with an 8-channel head coil. Each of these sessions included a 4-min T1weighted (T1w) 3D anatomical run (124 transverse slices, field of view $=256 \mathrm{~mm}^{3}$, vox $=0.8 \mathrm{~mm}^{3}$ ) and a 15-min T2*-weighted 3D-PRESTO sequence (36-38). This functional sequence (dynamic scan time $=1000 \mathrm{~ms}, \mathrm{TE}=9.6 \mathrm{~ms}$, flip angle $=9^{\circ}$, vox $=3.3 \mathrm{~mm}^{3}$ ) allowed for full functional brain coverage with a temporal resolution particularly suited for effective connectivity analysis (39).

Anatomical and functional MRI data were preprocessed using the FMRIPrep pipeline v. 1.5 (40), a Nipype v. 1.2.2 based tool (41). T1w images were corrected for nonuniform intensity and skull stripped. Brain tissue segmentation of cerebrospinal fluid, white-matter and gray-matter was performed on the brain-extracted T1w image. Volume-based spatial normalization to the Montreal Neurological Institute ICBM-152 (MNI) template was performed through nonlinear registration using brain-extracted versions of both the T1w reference and the MNI template.

For functional images, a reference BOLD volume and its skull-stripped version were generated and coregistered to the T1w image using a boundary-based registration algorithm with 9 degrees of freedom. Head-motion parameters were estimated before spatiotemporal filtering. Motion correction, BOLD-to-T1w transformation and T1w-to-template (MNI) warps were concatenated and applied in a single step using antsApplyTransforms (ANTs v2.1.0) based on Lanczos interpolation. Spatial smoothing with a 6-mm isotropic Gaussian kernel was then performed, and a 
second "nonaggressive" denoising step was conducted using independent component analysis [ICAAROMA]. Linear trends were finally removed, and a high-pass temporal filtering with 3 cycles/point was applied.

\section{Granger causality analysis}

Effective connectivity was assessed using Granger causality analysis (GCA), which allows for the data-driven exploration of a reference region's influence over the brain as well as targets of influence on the same given area (42). We used this approach to account for the multiple regions naturally connected to the SN (43) and to avoid missing brain areas that could not have been retained in a more traditional theory-driven framework. Here, we used the implementation proposed in the BrainVoyager software suite (v21.4, BrainInnovation, Maastricht; Granger Causality Mapping plugin v1.5). We defined the bilateral Al, according to the meta-analysis from Laird et al. (Figure 1) (2011) (44), as the reference region for later analyses. GCA maps were used to visualize the directed influences between the Al and every voxel in both directions after applying a graymatter mask. These maps were thresholded using a false-discovery rate approach at q-levels of 0.01 . We finally tested the association between GCA maps and symptom severity at the V7 time-point, considering $p_{\text {fwe }}<0.05$ as significant.

\section{$\underline{\text { Main statistical analysis }}$}

To assess changes in Al effective-connectivity pre/post treatment between patients who respond to treatment (responders) and those who did not (nonresponders), we referred to a $2 \times 2$ mixed-model ANOVA, using GCA maps at V1 and V7 as within-subject factors and treatment response as a between-subject factor. Post-hoc analyses used Student's t-tests. All maps were then thresholded using a cluster-based permutation method (45). To prevent potential inflated falsepositive rates (46), we first specified a cluster-defining threshold $(C D T)$ at $p_{\text {uncorrected }}<0.001$. After conducting a 1000-iteration Monte-Carlo simulation, a cluster-extent threshold was defined as a 
value high enough to keep the family-wise error at $p_{f w e}=0.05$. The resulting brain areas were labeled using the Anatomy toolbox v 3.0 (https://github.com/inm7/jubrain-anatomy-toolbox, (47)).

\section{$\underline{\text { Intrinsic network spatial stability measure }}$}

In parallel, we also explored the spatial stability of the DMN and the CEN posttreatment (i.e., the V7 time-point) using a "goodness-of-fit" (GoF) procedure. After decomposing each posttreatment functional dataset using independent component analysis (ICA), we selected the components exhibiting the highest spatial correlation with an a priori template. For each participant, this procedure was repeated twice : with the DMN and the CEN template, respectively (48). The resulting GoF scores were assumed to reflect posttreatment DMN and CEN spatial stability. We tested for an association between these GoF scores and the severity of intrusive symptoms using Pearson's $r$ correlation test, considering $\mathrm{p}<0.05$ as significant. 


\section{RESULTS}

\section{Demographic and clinical variables}

At baseline, responders and nonresponders were comparable in terms of (i) sociodemographic characteristics (age and sex-ratio), and (ii) symptom severity (PCL-S scores/subscores and depressive symptoms measured with the Beck Depression Inventory (49)). Note that, counterintuitively, the responder group contained fewer patients receiving propranolol (see (31) for a more detailed description of the RCT main findings).

Table 1: Population description

\begin{tabular}{|c|c|c|}
\hline & Responders ( $n=16)$ & Nonresponders $(n=14)$ \\
\hline Age & $41.3(12.3)$ & $36.6(13.5)$ \\
\hline Sex (Male /Female) & $7 / 9$ & $8 / 6$ \\
\hline Dvars & $24.3(3.74)$ & $24.1(4.05)$ \\
\hline Global signal & $887(121)$ & $916(96.3)$ \\
\hline Total PCL-S score & $65.4(9.34)$ & $68.4(9.34)$ \\
\hline PCLS-score (question 1 to 5 ) & $18.9(3.38)$ & $19.4(3.38)$ \\
\hline $\begin{array}{l}\text { Beck Depression inventory } \\
\text { score }\end{array}$ & $25.9(14.5)$ & $28.9(11.3)$ \\
\hline $\begin{array}{l}\text { Rate of patients receiving } \\
\text { propranolol * }\end{array}$ & $31.3 \%$ & $78.6 \%$ \\
\hline
\end{tabular}




\section{INSERT FIGURE 1 ABOUT HERE}

\section{Al effective connectivity at V1}

GCA performed on the whole-sample at baseline revealed that the bilateral Al significantly modulates a group of regions involved in motor preparation, execution and action monitoring (i.e., precentral gyrus, the supplementary motor area, the left thalamus, and the frontal pole), as well as in visuospatial processing (i.e., the paracingulate gyrus and the precuneus). See Figure 1 and Suppl.

Table 2. No specific connectivity differences were evidenced between the responder and nonresponder groups before treatment.

\section{Al effective-connectivity changes between V1 and V7}

The mixed-model ANOVA revealed significant changes in Al causal maps after treatment. A significant [time-point $x$ group] interaction was evidenced: (i) laterally, in the mid- and posterior insula, amygdala, precentral gyrus and supramarginal gyrus; and (ii) medially, in the cingulate cortex (anterior and posterior) and the precuneus. Compared with nonresponders, patients showing clinical improvement exhibited a reduced influence of the Al over a wide socioemotional network composed of the superior frontal gyrus, anterior and posterior supramarginal gyri, anterior and posterior cingulate, central operculum and right amygdala. Conversely, responders exhibited a higher influence of the Al over the precuneus (Cf. Figure 2, Table 2). Simple pre/posttreatment contrasts for responders and nonresponders are available in Suppl. Figures 1, 2 and Suppl. Tables 3, 4 respectively, As a point of comparison, significant results for propranolol vs placebo at V7 are presented in Suppl. Figure 3 and Suppl. Table 5.

Regression analysis conducted posttreatment further revealed that the more severe the intrusive symptoms were, the greater the Al exerted influence over somatosensory and motor regions (i.e., the posterior insula, right parietal operculum, and precentral gyrus), as well as over brain areas involved in visuospatial processing (the paracingulate gyrus) and self-other processing 
(i.e., the anterior and posterior cingulate cortices, superior frontal gyrus and supramarginal gyrus).

See Figure 3.

Table 2: Changes in the anterior insula effective connectivity between responder and nonresponder patients. Regions exhibiting a significant difference in Granger causality analysis maps between V1 and V7 according to treatment response. Coordinates are reported in MNI (Montreal Neurological Institute) space.

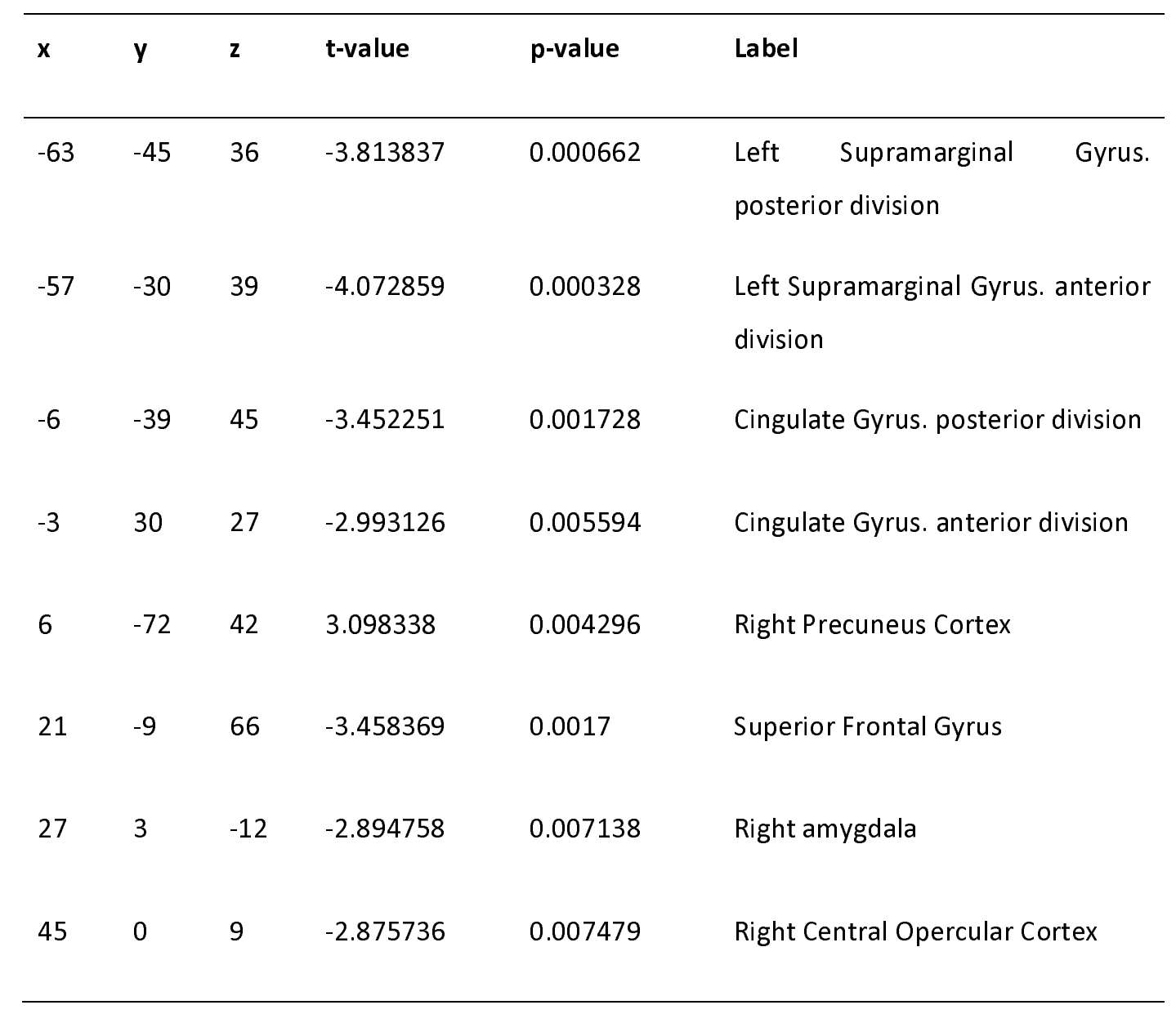




\section{$\underline{\text { Intrinsic network spatial stability measure at V7 }}$}

Finally, the assessment of the spatial stability of the DMN and CEN posttreatment revealed that the severity of "re-experiencing" symptoms was positively correlated with the DMN GoF scores $(r=0.521, p=0.003)$ and negatively correlated with the CEN GoF scores $(r=-0.418 p=0.021-C f$.

\section{Figure 3).}




\section{DISCUSSION}

The present fMRI study was designed to explore how an effective response to traumatic memory reactivation therapy (with or without propranolol) for intrusive symptoms in PTSD could modulate Al causal influences over the brain. Very limited fMRI studies measured pre/post treatment changes in PTSD (50) and to our knowledge, none of them included effective connectivity analysis. We focused on the Al since this region is known to be: (i) a central integration hub serving sensory, emotional, motivational and cognitive functions, (ii) under B-noradrenergic regulation, and (iii) potentially involved in re-experiencing trauma. Using high temporal-resolution $\mathrm{FMRI}$ and comparing pre/post therapy GCA results in PTSD patients, we were able to provide evidence that treatment response was associated with a significant reduction in Al effective connectivity towards motor and socioaffective regions, a global decrease that follows symptom severity reduction.

The first set of regions modulated by Al corresponded to limbic areas for which dense reciprocal connections with the ventral Al were repeatedly described. A strong body of evidence supports Al mediation in fear and anxiety, which was regularly found to be coactivated with the amygdala in stressful contexts (9). By showing a reduced influence of Al on the amygdala in responders compared with nonresponders, we can assume that one of the first effects of treatment was to temper the emotional storm associated with re-experiencing and hyperarousal [18]. This finding nicely complements the existing literature in which joint amygdalar and insular overactivation is described in the context of PTSD (20) and declines after successful therapy (51). The ability to better modulate Al connectivity following therapy could be associated with better cross-talk between untargeted inner thoughts and the ability to focus attention on stimulus-dependent demands (52), a theory also supported by the association found between symptom severity and $\mathrm{Al}$ influence over visuospatial areas. Using GCA, we were able to attest that Al primarily drives this pathological interaction in PTSD. 
The second set of brain areas modulated by Al are involved in self-other distinction and may support dissociative experiences frequently observed in PTSD. This is the case for the precuneus, frontal superior and supramarginal gyri, all regularly found to be involved in self-awareness and agency processing (e.g., (53)). These cognitive functions are more usually under the influence of the dorsal Al (54). Interestingly, localized Al lesions can induce dissociative experiences, such as the (rare) "pain asymbolia" syndrome, in which pain recognition appears disconnected from its appropriate emotional response (9). Within this functional network, the supramarginal gyrus, located at the temporo-parietal junction, has also been linked with experiences involving a sensorial component. Similar to the Al, the supramarginal gyrus receives heavy sensory inputs ranging from the auditory to the somatosensory modality. The crossmodal nature of this area makes it particularly well suited for linking sensory experiences with cognitive and/or affective information. Finally, the supramarginal gyrus is also involved in the phonological and articulatory processing of words (55), making it solicistable by talking therapy. Again, this is perfectly in line with the present findings showing that the Al influence on this network was correlated with the degree of intrusion and was significantly decreased in responders.

The third set of regions influenced by $\mathrm{Al}$ is engaged in sensorimotor control (56-58) and might be involved in the autonomic and behavioral responses to stress. Again, this interaction was found to correlate with symptom severity, even if only indirectly through the posterior insula and thalamic relays (59), which were found to be under Al control at baseline. The motor network under consideration includes the caudal anterior cingulate cortex (CACC), the supplementary motor area and the precentral gyrus. A decreased resting-state functional connectivity between the CACC and the precentral gyrus was previously evidenced in veterans with or without PTSD compared to healthy controls, suggesting that military training or deployment, including trauma exposure, may influence SN connectivity (60). In addition, precentral activity has been related to defensive behaviors in animals (61) and may subserve the increased "fight or flight" response regularly observed in PTSD when facing mental stress. 
In addition to networks sustaining the rich phenomenology of PTSD symptoms, we also investigated how effective treatment may dynamically affect the interaction between intrinsic neural networks. We first demonstrated that the more the Al exerted a causal influence over core nodes of the DMN (such as the rostral ACC, the posterior cingulate and the precuneus), the more severe intrusive symptoms were, and that this interaction differentially changed at V7 according to the treatment response. This finding appears in line with previous studies conducted in schizophrenia patients (21), showing that increased control from the SN to the DMN initiates hallucinatory states. Interestingly, the DMN is also known to anti-correlate with task-related networks, such as the CEN $(62,63)$, and this antagonistic activity was proposed to be tuned by the Al $(23)$. Returning to our schizophrenia example, a CEN takeover was found to drive the extinction of hallucinations (21). Here, we found that re-experiencing the trauma positively correlated with DMN stability and presented a reversed pattern for the CEN. Altogether, these results support the idea that intrusive symptoms could correspond to self-referential mental activities driven by impaired Al control over the DMN/CEN balance, making salient memory fragments active enough (through bottom-up amplification) to aberrantly intrude into consciousness.

Despite these encouraging findings, some issues need to be further discussed. If the above mentioned theory is correct, we could expect to find the hippocampal complex among the regions influenced by the Al. The limited sample-size of the present trial may account for such a negative result (i.e., a power issue), and the exact relationship between the Al and this limbic structure will have to be clarified in future studies. In fact, several brain areas identified in this study have previously been shown to be involved in memory suppression beyond the medial temporal lobe. This is the case for the precuneus and the frontal cortex (64), which have tight connections with the hippocampus $(65,66)$. The same is true for other limbic structures, such as the amygdala which have strong reciprocal connections with the hippocampus. We cannot exclude that intermediate small brain structures, such as the hippocampus, that are involved in a chain of causality could be more vulnerable and may not survive statistical thresholding. Based on that possibility, we hypothesize a 
triple interaction [AI - amygdala - hippocampus] at the root of the memorization of trauma-related emotional valence, constituting an interesting complementary track for future research on PTSD.

A second potential issue resides in the fact that GCA indicates the dominant direction of influence, introducing ambiguity in the interpretation of pre/post treatment contrast maps, as they may potentially result from a decrease in the influence of Al-to-target-regions influence or from an increase in the influence of target-to-Al. This problem can, for instance, be illustrated by considering a recent study of effective connectivity in PTSD that reached seemingly opposite conclusions, suggesting that frontal regions exerted a reduced influence on $\mathrm{Al}(67)$. In the same vein, a decreased causal flow from the right amygdala to the right insula in PTSD patients relative to trauma-exposed controls has been suggested (24), which again appears in apparent contradiction with the present findings. Even if the samples and designs were not exactly comparable, methodological advances should help to reconcile these findings, but until then, this literature needs to be interpreted with caution and in reference to clinically and anatomo-functionally available knowledge at the time of publication.

A final point we would like to insist on is that we focused on symptom reduction, regardless of the initial group of randomisation in the Pre-Reactivation Propranolol Therapy trial. Of course, the full results of the RCT have been presented elsewhere (31) and are beyond the scope of the present paper. Note that the between-group differences in our study were both explained by changes in the responder and non-responder groups. This is compatible with previous studies showing that psychotherapy could induce functional changes, even in cases of no clinical response (50), that could be linked with repeated trauma exposure without extinction, as suggested in previous studies $(60)$. Importantly, because half of the sample did not reach the threshold for a positive response to therapy, we expect our findings to also be relevant for future neuromodulation trials for severely impaired PTSD patients. Neuromodulation methods such as transcranial magnetic stimulation usually targets superficial cortical areas, and Al could constitute a better target for neurofeedback. 
Promisingly, a recent study confirmed that PTSD patients could be trained to downregulate amygdalar activity using real time fMRI-based neurofeedback (68).

Overall, we were able to provide experimental support for the causal role played by Al in reexperiencing trauma. Notably, we showed that effective therapy was linked with plastic changes in Al directed influence over sensorimotor, cognitive and socioemotional networks. Dynamically, restoration of the DMN-to-CEN switch control was also observed, offering an attractive mechanism for intrusion. We hope that the present results will contribute to paving the way for new evidencedbased treatments of intrusive symptoms in PTSD, considering Al as a particularly interesting target for this purpose. 


\section{ACKNOWLEDGMENTS}

Funding source: French Ministry of Health's Hospital Program of Clinical Research

\section{DISCLOSURE}

AL is consultant for Kinnov Therapeutics. RJ has been invited to scientific meetings, and boards with compensation, by Lundbeck, Janssen and Otsuka. None of these links of interest are related to the present work. All the other authors have no conflict of interest to declare. 


\section{REFERENCES}

1. Horesh D, Brown AD (2020): Traumatic stress in the age of COVID-19: A call to close critical gaps and adapt to new realities. Psychol Trauma Theory Res Pract Policy 12: 331-335.

2. Lewis SJ, Arseneault L, Caspi A, Fisher HL, Matthews T, Moffitt TE, et al. (2019): The epidemiology of trauma and post-traumatic stress disorder in a representative cohort of young people in England and Wales. Lancet Psychiatry 6: 247-256.

3. American Psychiatric Association (1994): Diagnostic and Statistical Manual of Mental Disorders, Fourth Edition. American Psychiatric Association.

4. Brewin CR, Gregory JD, Lipton M, Burgess N (2010): Intrusive images in psychological disorders: characteristics, neural mechanisms, and treatment implications. Psychol Rev 117: 210-232.

5. Larøi F, Sommer IE, Blom JD, Fernyhough C, Ffytche DH, Hugdahl K, et al. (2012): The characteristic features of auditory verbal hallucinations in clinical and nonclinical groups: state-of-the-art overview and future directions. Schizophr Bull 38: 724-733.

6. Newman MG, Llera SJ, Erickson TM, Przeworski A, Castonguay LG (2013): Worry and generalized anxiety disorder: a review and theoretical synthesis of evidence on nature, etiology, mechanisms, and treatment. Annu Rev Clin Psychol 9: 275-297.

7. Watkins ER (2008): Constructive and unconstructive repetitive thought. Psychol Bull 134: 163-206.

8. van den Heuvel MP, Sporns O (2013): Network hubs in the human brain. Trends Cogn Sci 17: 683696.

9. Gogolla N (2017): The insular cortex. Curr Biol CB 27: R580-R586.

10. Augustine JR (1996): Circuitry and functional aspects of the insular lobe in primates including humans. Brain Res Rev 22: 229-244.

11. Bamiou D-E, Musiek FE, Luxon LM (2003): The insula (Island of Reil) and its role in auditory processing: Literature review. Brain Res Rev 42: 143-154.

12. Butti C, Hof PR (2010): The insular cortex: a comparative perspective. Brain Struct Funct 214: 477-493. 
13. Mesulam M-M, Mufson EJ (1982): Insula of the old world monkey. Architectonics in the insuloorbito-temporal component of the paralimbic brain. J Comp Neurol 212: 1-22.

14. Nieuwenhuys R (2012): Chapter 7 - The insular cortex: A review. In: Hofman MA, Falk D, editors. Progress in Brain Research, vol. 195. Elsevier, pp 123-163.

15. Bushara, Grafman J, Hallett M (2001): Neural correlates of auditory-visual stimulus onset asynchrony detection. J Neurosci Off J Soc Neurosci 21: 300-304.

16. Bushara, Hanakawa T, Immisch I, Toma K, Kansaku K, Hallett M (2003): Neural correlates of crossmodal binding. Nat Neurosci 6: 190-195.

17. Menon, Uddin LQ (2010): Saliency, switching, attention and control: a network model of insula function. Brain Struct Funct 214: 655-667.

18. Yehuda R, Hoge CW, McFarlane AC, Vermetten E, Lanius RA, Nievergelt CM, et al. (2015): Posttraumatic stress disorder. Nat Rev Dis Primer 1: 15057.

19. Stevens JS, Reddy R, Kim YJ, van Rooij SJH, Ely TD, Hamann S, et al. (2018): Episodic memory after trauma exposure: Medial temporal lobe function is positively related to re-experiencing and inversely related to negative affect symptoms. Neurolmage Clin 17: 650-658.

20. Koch SBJ, van Zuiden M, Nawijn L, Frijling JL, Veltman DJ, Olff M (2016): Aberrant resting-state brain activity in posttraumatic stress disorder: a meta-analysis and systematic review. Depress Anxiety 33: 592-605.

21. Lefebvre S, Demeulemeester M, Leroy A, Delmaire C, Lopes R, Pins D, et al. (2016): Network dynamics during the different stages of hallucinations in schizophrenia. Hum Brain Mapp 37: 2571-2586.

22. Palaniyappan L, Liddle PF (2012): Does the salience network play a cardinal role in psychosis? An emerging hypothesis of insular dysfunction. J Psychiatry Neurosci JPN 37: 17-27.

23. Sridharan D, Levitin DJ, Menon V (2008): A critical role for the right fronto-insular cortex in switching between central-executive and default-mode networks. Proc Natl Acad Sci 105: $12569-12574$. 
24. Weng Y, Qi R, Zhang L, Luo Y, Ke J, Xu Q, et al. (2019): Disturbed effective connectivity patterns in an intrinsic triple network model are associated with posttraumatic stress disorder. Neurol Sci Off J Ital Neurol Soc Ital Soc Clin Neurophysiol 40: 339-349.

25. Zerbi V, Floriou-Servou A, Markicevic M, Vermeiren Y, Sturman O, Privitera M, et al. (2019): Rapid Reconfiguration of the Functional Connectome after Chemogenetic Locus Coeruleus Activation. Neuron 103: 702-718.e5.

26. Onur OA, Walter H, Schlaepfer TE, Rehme AK, Schmidt C, Keysers C, et al. (2009): Noradrenergic enhancement of amygdala responses to fear. Soc Cogn Affect Neurosci 4: 119-126.

27. Cameron OG, Minoshima S (2002): Regional brain activation due to pharmacologically induced adrenergic interoceptive stimulation in humans. Psychosom Med 64: 851-861.

28. Hermans EJ, van Marle HJF, Ossewaarde L, Henckens MJAG, Qin S, van Kesteren MTR, et al. (2011): Stress-related noradrenergic activity prompts large-scale neural network reconfiguration. Science 334: 1151-1153.

29. Cousijn H, Rijpkema M, Qin S, van Marle HJF, Franke B, Hermans EJ, et al. (2010): Acute stress modulates genotype effects on amygdala processing in humans. Proc Natl Acad Sci U S A 107: 9867-9872.

30. Brunet A, Saumier D, Liu A, Streiner DL, Tremblay J, Pitman RK (2018): Reduction of PTSD Symptoms With Pre-Reactivation Propranolol Therapy: A Randomized Controlled Trial. Am J Psychiatry 175: 427-433.

31. Roullet P, Vaiva G, Véry E, Bourcier A, Yrondi A, Dupuch L, et al. (submitted): Traumatic memory reactivation with or without propranolol for PTSD and comorbid MD symptoms: A randomized clinical trial.

32. Ventureyra VAG, Yao S-N, Cottraux J, Note I, De Mey-Guillard C (2002): The validation of the Posttraumatic Stress Disorder Checklist Scale in posttraumatic stress disorder and nonclinical subjects. Psychother Psychosom 71: 47-53. 
33. Thierrée S, Richa S, Brunet A, Egreteau L, Roig Q, Clarys D, El-Hage W (2020): Trauma reactivation under propranolol among traumatized Syrian refugee children: preliminary evidence regarding efficacy. Eur J Psychotraumatology 11: 1733248.

34. Brady F, Warnock-Parkes E, Barker C, Ehlers A (2015): Early in-session predictors of response to trauma-focused cognitive therapy for posttraumatic stress disorder. Behav Res Ther 75: 4047.

35. Mushtaq D, Ali A, Margoob MA, Murtaza I, Andrade C (2012): Association between serotonin transporter gene promoter-region polymorphism and 4- and 12-week treatment response to sertraline in posttraumatic stress disorder. J Affect Disord 136: 955-962.

36. Liu G, Sobering G, Duyn J, Moonen CT (1993): A functional MRI technique combining principles of echo-shifting with a train of observations (PRESTO). Magn Reson Med 30: 764-768.

37. Neggers SFW, Hermans EJ, Ramsey NF (2008): Enhanced sensitivity with fast three-dimensional blood-oxygen-level-dependent functional MRI: comparison of SENSE-PRESTO and 2D-EPI at 3 T. NMR Biomed 21: 663-676.

38. van Gelderen P, Duyn JH, Ramsey NF, Liu G, Moonen CTW (2012): The PRESTO technique for fMRI. Neurolmage 62: 676-681.

39. Deshpande G, Hu X (2012): Investigating effective brain connectivity from fMRI data: past findings and current issues with reference to Granger causality analysis. Brain Connect 2: $235-245$.

40. Esteban O, Markiewicz CJ, Blair RW, Moodie CA, Isik Al, Erramuzpe A, et al. (2019): fMRIPrep: a robust preprocessing pipeline for functional MRI. Nat Methods 16: 111-116.

41. Gorgolewski K, Burns CD, Madison C, Clark D, Halchenko YO, Waskom ML, Ghosh SS (2011):

Nipype: a flexible, lightweight and extensible neuroimaging data processing framework in python. Front Neuroinformatics 5: 13.

42. Roebroeck A, Formisano E, Goebel R (2005): Mapping directed influence over the brain using Granger causality and fMRI. Neurolmage 25: 230-242. 
43. Menon (2015): Salience Network. In: Toga AW, editor. Brain Mapping. Waltham: Academic Press, pp 597-611.

44. Laird AR, Fox PM, Eickhoff SB, Turner JA, Ray KL, McKay DR, et al. (2011): Behavioral interpretations of intrinsic connectivity networks. J Cogn Neurosci 23: 4022-4037.

45. Forman SD, Cohen JD, Fitzgerald M, Eddy WF, Mintun MA, Noll DC (1995): Improved assessment of significant activation in functional magnetic resonance imaging (fMRI): use of a cluster-size threshold. Magn Reson Med 33: 636-647.

46. Eklund A, Nichols TE, Knutsson H (2016): Cluster failure: Why fMRI inferences for spatial extent have inflated false-positive rates. Proc Natl Acad Sci U S A 113: 7900-7905.

47. Eickhoff SB, Stephan KE, Mohlberg H, Grefkes C, Fink GR, Amunts K, Zilles K (2005): A new SPM toolbox for combining probabilistic cytoarchitectonic maps and functional imaging data. Neurolmage 25: 1325-1335.

48. Greicius MD, Srivastava G, Reiss AL, Menon V (2004): Default-mode network activity distinguishes Alzheimer's disease from healthy aging: evidence from functional MRI. Proc Natl Acad Sci US A 101: 4637-4642.

49. Beck AT, Steer RA, Carbin MG (1988): Psychometric properties of the Beck Depression Inventory: Twenty-five years of evaluation. Clin Psychol Rev 8: 77-100.

50. Simmons AN, Norman SB, Spadoni AD, Strigo IA (2013): Neurosubstrates of remission following prolonged exposure therapy in veterans with posttraumatic stress disorder. Psychother Psychosom 82: 382-389.

51. Malejko K, Abler B, Plener PL, Straub J (2017): Neural Correlates of Psychotherapeutic Treatment of Post-traumatic Stress Disorder: A Systematic Literature Review. Front Psychiatry 8: 85.

52. Szeszko PR, Yehuda R (2019): Magnetic resonance imaging predictors of psychotherapy treatment response in post-traumatic stress disorder: A role for the salience network. Psychiatry Res 277: 52-57. 
53. Sperduti M, Delaveau P, Fossati P, Nadel J (2011): Different brain structures related to self- and external-agency attribution: a brief review and meta-analysis. Brain Struct Funct 216: 151157.

54. Kurth F, Zilles K, Fox PT, Laird AR, Eickhoff SB (2010): A link between the systems: functional differentiation and integration within the human insula revealed by meta-analysis. Brain Struct Funct 214: 519-534.

55. Stoeckel C, Gough PM, Watkins KE, Devlin JT (2009): Supramarginal gyrus involvement in visual word recognition. Cortex J Devoted Study Nerv Syst Behav 45: 1091-1096.

56. Chouinard PA, Paus T (2006): The primary motor and premotor areas of the human cerebral cortex. Neurosci Rev J Bringing Neurobiol Neurol Psychiatry 12: 143-152.

57. Dum RP, Strick PL (1991): The origin of corticospinal projections from the premotor areas in the frontal lobe. J Neurosci Off J Soc Neurosci 11: 667-689.

58. Paus T (2001): Primate anterior cingulate cortex: where motor control, drive and cognition interface. Nat Rev Neurosci 2: 417-424.

59. Uddin LQ, Nomi JS, Hebert-Seropian B, Ghaziri J, Boucher O (2017): Structure and function of the human insula. J Clin Neurophysiol Off Publ Am Electroencephalogr Soc 34: 300.

60. Kennis M, Rademaker AR, van Rooij SJH, Kahn RS, Geuze E (2014): Resting state functional connectivity of the anterior cingulate cortex in veterans with and without post-traumatic stress disorder. Hum Brain Mapp 36: 99-109.

61. Graziano MSA, Cooke DF (2006): Parieto-frontal interactions, personal space, and defensive behavior. Neuropsychologia 44: 845-859.

62. Fox MD, Snyder AZ, Vincent JL, Corbetta M, Van Essen DC, Raichle ME (2005): The human brain is intrinsically organized into dynamic, anticorrelated functional networks. Proc Natl Acad Sci U S A 102: 9673-9678.

63. Greicius MD, Krasnow B, Reiss AL, Menon V (2003): Functional connectivity in the resting brain: a network analysis of the default mode hypothesis. Proc Natl Acad Sci U S A 100: 253-258. 
64. Mary A, Dayan J, Leone G, Postel C, Fraisse F, Malle C, et al. (2020): Resilience after trauma: The role of memory suppression. Science 367 . https://doi.org/10.1126/science.aay8477

65. Cunningham SI, Tomasi D, Volkow ND (2017): Structural and functional connectivity of the precuneus and thalamus to the default mode network. Hum Brain Mapp 38: 938-956.

66. Anderson MC, Bunce JG, Barbas H (2016): Prefrontal-hippocampal pathways underlying inhibitory control over memory. Neurobiol Learn Mem 134 Pt A: 145-161.

67. Rangaprakash D, Dretsch MN, Venkataraman A, Katz JS, Denney TS, Deshpande G (2018):

Identifying disease foci from static and dynamic effective connectivity networks: Illustration in soldiers with trauma. Hum Brain Mapp 39: 264-287.

68. de Pierrefeu A, Fovet T, Hadj-Selem F, Löfstedt T, Ciuciu P, Lefebvre S, et al. (2018): Prediction of activation patterns preceding hallucinations in patients with schizophrenia using machine learning with structured sparsity. Hum Brain Mapp 39: 1777-1788. 


\section{FIGURES \& TABLES}

Figure 1. Study design and Granger causality analysis (GCA) seeded on the anterior insula in PTSD patients. (A) The bilateral anterior insula was chosen as the region of interest for GCA and is presented in red in a glass brain. (B) Flow chart of the study. We defined responders as patients with at least a 33\% decrease in PCL-S scores at V7 compared with baseline (V1). (C) Whole-sample random-effects GCA map at V1. PCL-S: Posttraumatic Stress Disorder Checklist Scale; Thal: thalamus; Hipp: hippocampus; SMA: supplementary motor area; A: anterior; P: posterior; L/R: left/right sides of the brain.

Figure 2. Changes in Granger causality maps seeded on the anterior insula (AI) between responders and non-responders to therapy in PTSD. We used a transparent right hemisphere to allow visualization of the deeper clusters. Brain areas less influenced by Al after effective treatment are depicted in dark blue. The precuneus (pink) was the only cluster found to be more influenced by Al posttreatment in responders than in non-responders.

Figure 3. Brain correlates of intrusive symptom severity in PTSD. (A) Linear regression analysis showing the brain regions exhibiting a positive association between the severity of intrusive symptoms and thresholded Granger causality analysis maps at V7. (B, C) Correlation analyses between intrinsic network stability and the severity of intrusive symptoms at V7. A positive association was indicated by the default mode network stability (DMN GoF score), shown in (B), whereas a negative association was indicated by the central executive network stability (CEN GoF score), shown in (C).

\section{Table 1: Population description.}

Table 2: Changes in the anterior insula effective connectivity between responder and nonresponder patients. Regions exhibiting a significant difference in Granger causality analysis maps between V1 and V7 according to treatment response. Coordinates are reported in the MNI (Montreal Neurological Institute) space. 

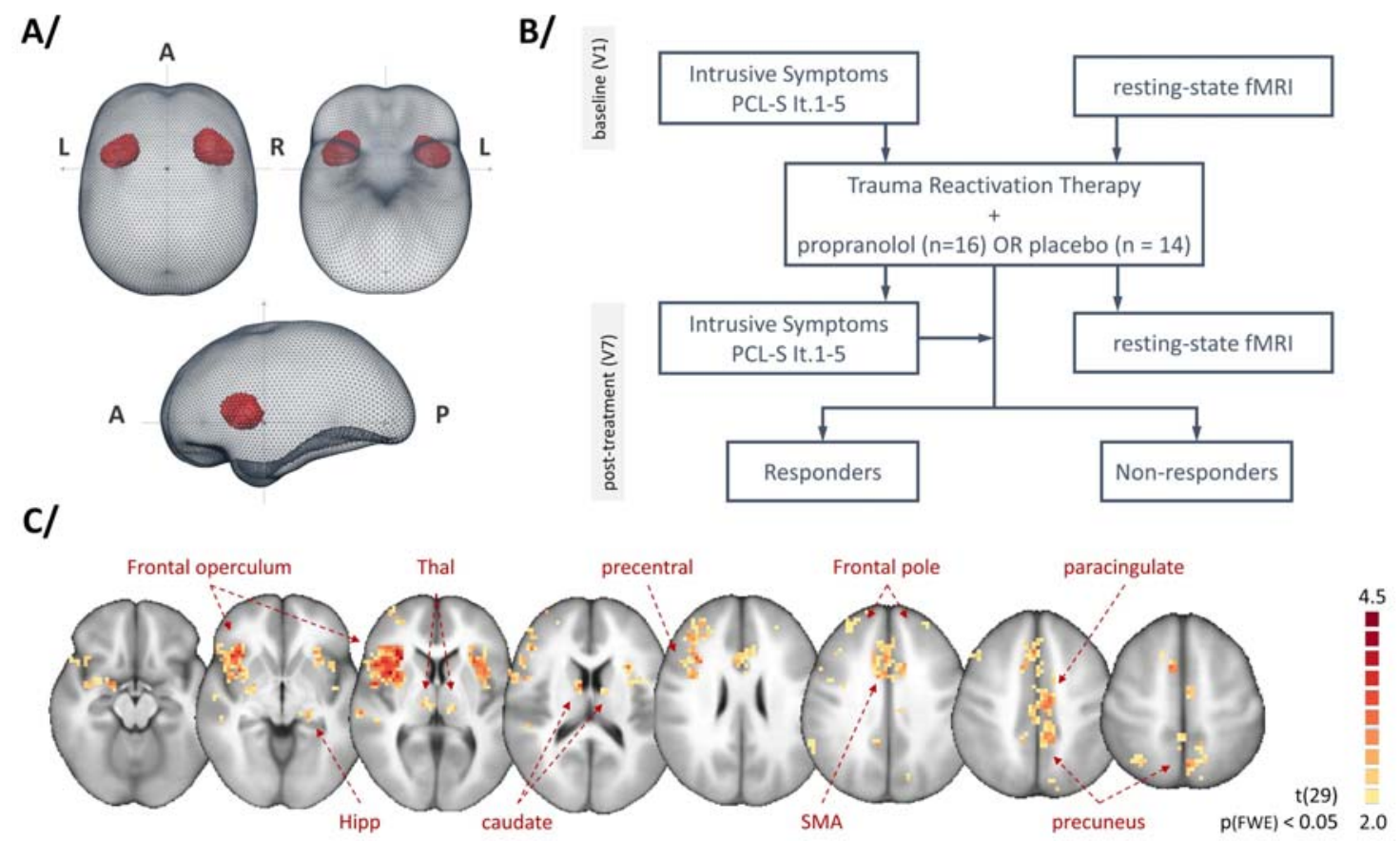

Figure 1. Study design and Granger causality analysis (GCA) seeded on the anterior insula in PTSD patients. (A) The bilateral anterior insula was chosen as the region of interest for GCA and is presented in red in a glass brain. (B) Flow chart of the study. We defined responders as patients with at least a 33\% decrease in PCL-S scores at V7 compared with baseline (V1). (C) Whole-sample random-effects GCA map at V1. PCL-S: Posttraumatic Stress Disorder Checklist Scale; Thal: thalamus; Hipp: hippocampus; SMA: supplementary motor area; A: anterior; P: posterior; L/R: left/right sides of the brain. 


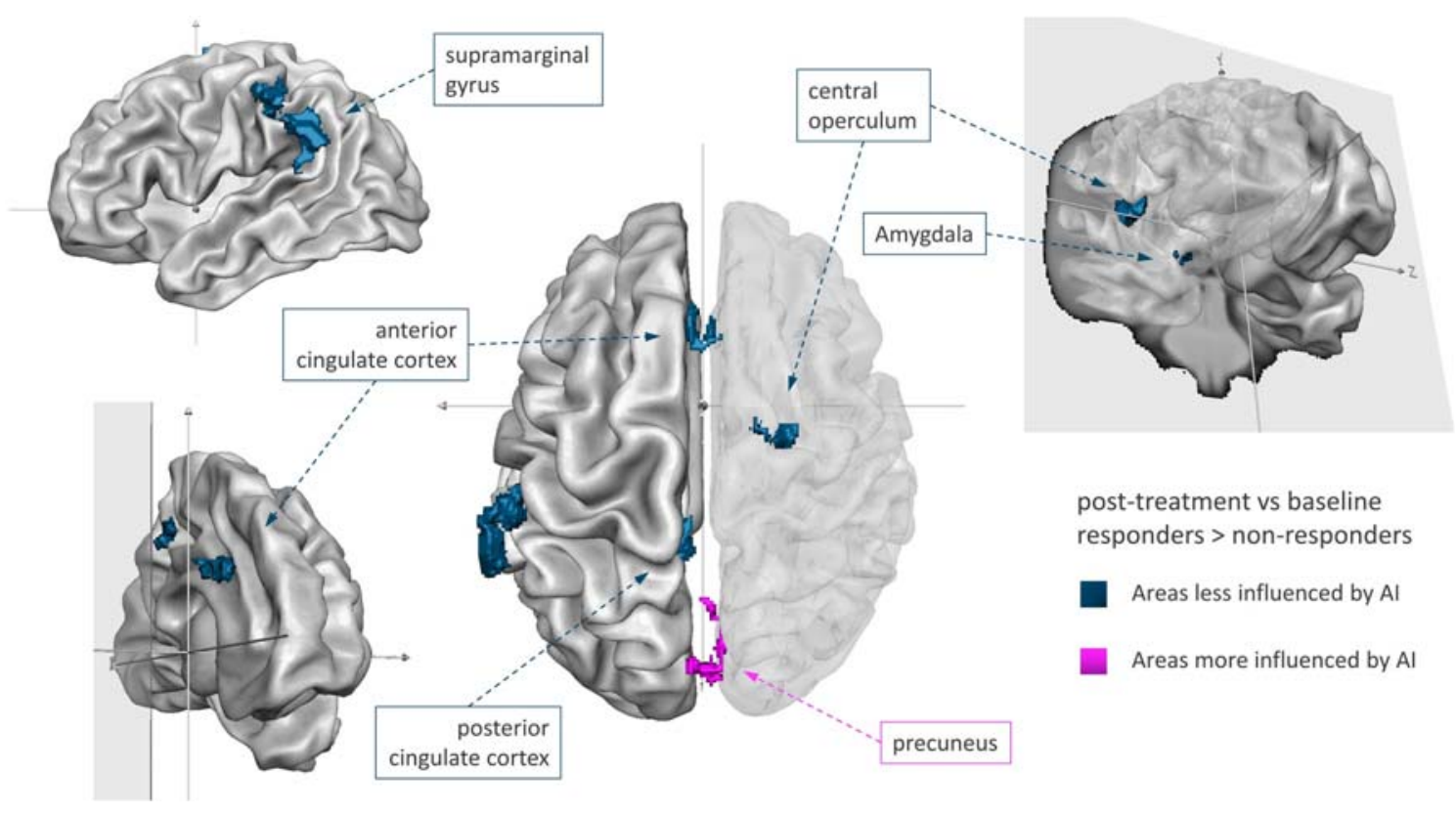

Figure 2. Changes in Granger causality maps seeded on the anterior insula (AI) between responders and non-responders to therapy in PTSD. We used a transparent right hemisphere to allow visualization of the deeper clusters. Brain areas less influenced by Al after effective treatment are depicted in dark blue. The precuneus (pink) was the only cluster found to be more influenced by Al posttreatment in responders than in non-responders. 

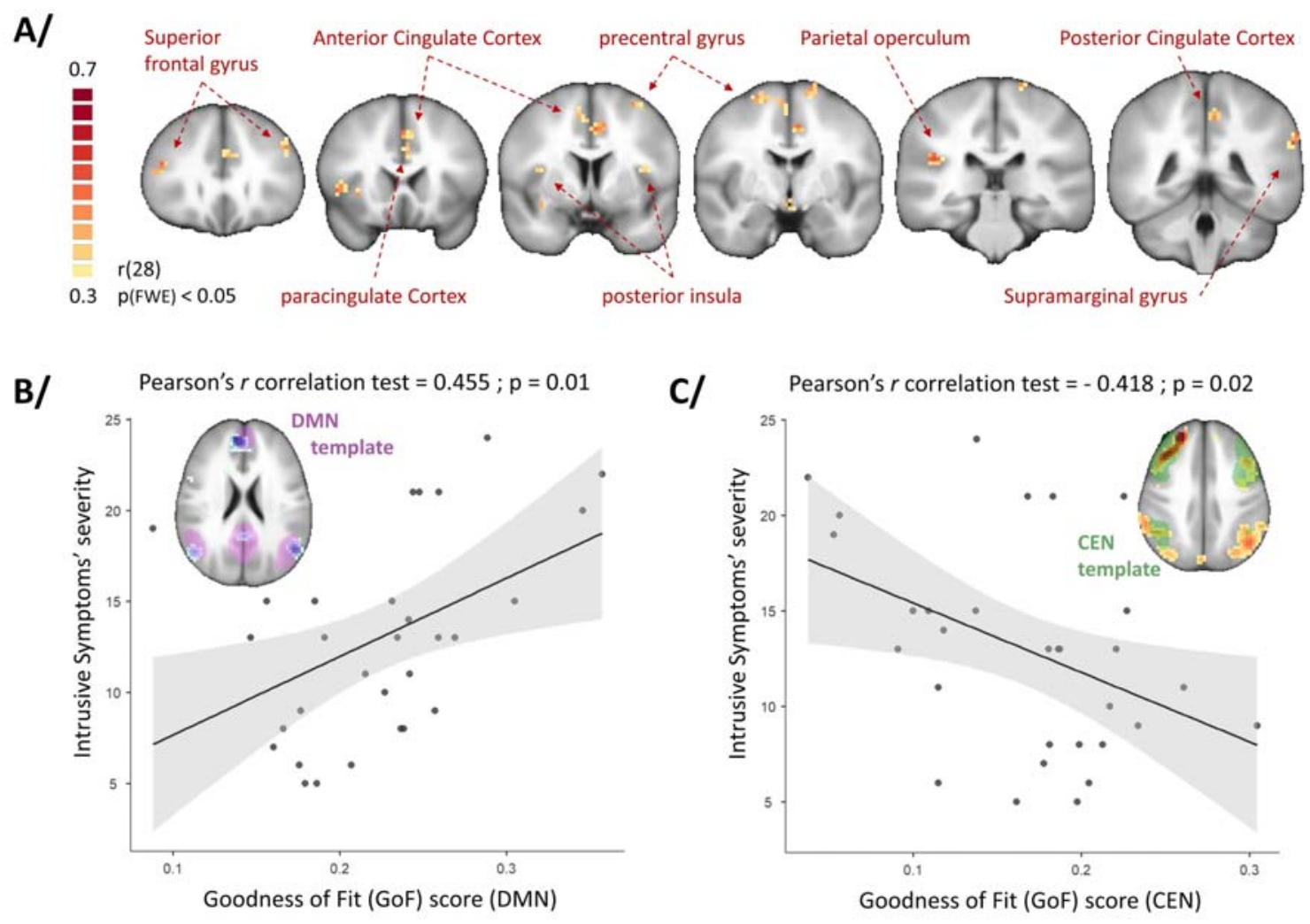

Figure 3. Brain correlates of intrusive symptom severity in PTSD. (A) Linear regression analysis showing the brain regions exhibiting a positive association between the severity of intrusive symptoms and thresholded Granger causality analysis maps at V7. (B, C) Correlation analyses between intrinsic network stability and the severity of intrusive symptoms at V7. A positive association was indicated by the default mode network stability (DMN GoF score), shown in (B), whereas a negative association was indicated by the central executive network stability (CEN GoF score), shown in (C). 